\title{
Nrp1, a Neuronal Regulator, Enhances DDR2-ERK-Runx2 Cascade in Osteoblast Differentiation via Suppression of DDR2 Degradation
}

\author{
Yan Zhang ${ }^{\mathrm{a}}$ Jin Su Yue Teng $^{\mathrm{a}}$ Jian Zhang ${ }^{\mathrm{a}}$ Jiang Wang ${ }^{\mathrm{c}}$ Kun Li ${ }^{\mathrm{d}}$ Libo Yao \\ $\mathrm{Xu} \mathrm{Li}{ }^{\mathrm{a}}$ \\ aCenter for Translational Medicine, The First Affiliated Hospital of Xi'an Jiaotong University College of \\ Medicine, ${ }^{b}$ Department of Biochemistry and Molecular Biology, The Fourth Military Medical University, \\ 'Department of Neurosurgery, Xijing Hospital, The Fourth Military Medical University, ${ }^{\mathrm{d} C l i n i c a l}$ \\ laboratory, Shanxi Mineral Hospital. Xi'an, People's Republic of China
}

\section{Key Words}

Neuropilin 1 • Discoidin domain receptor $2 \cdot$ Osteoblast differentiation

\begin{abstract}
Background: Osteoblastogenesis is under delicate control by multiple factors and hormones. Recent reports indicated the involvement of immunological and neuronal regulators. However, the role of neuropilin 1 (Nrp1) in osteoblastogenesis remains obscure. Methods: Real-time PCR was carried out to detect the mRNA of osteoblastic markers, Nrp1, and discoidin domain receptor 2 (DDR2). Immunoblot was performed to test the protein of Nrp1 and DDR2. Osteogenic differentiation was evaluated by mRNA analysis of osteogenic markers, and determination of ALP activity and OCN secretion. The intercellular signaling effectors were examined by immunoblot. Immunofluorescent assays were performed to detect the localization of Nrp1 and DDR2. Half-life determination assay was executed to test the DDR2 stability. Results: The expression of Nrp1 paralleled with that of DDR2 during osteoblastogensis. Nrp1 overexpression enhanced DDR2-induced stimulation of osteoblastogensis, whereas Nrp1 silencing caused attenuation. Nrp1 overexpression increased the phosphorylation of DDR2, ERK1/2 and Runx2. Nrp1 co-localized with DDR2 in the cellular membrane of differentiated MC3T3-E1. Enhanced or attenuated Nrp1 expression did not alter the mRNA transcript of DDR2. Nrp1 overexpression prolonged the half-life of DDR2 protein. Conclusion: Our results originally demonstrated the stimulatory role of Nrp1 in DDR2-induced osteoblast differentiation, providing molecular evidence for exploiting Nrp1 and DDR2 as targets to treat bone-related disease.
\end{abstract}


Zhang et al.: Nrp1 Augments DDR2-Mediated Signaling in Osteoblastogenesis

\section{Introduction}

Postnatal skeletal growth and bone remodeling are highly coordinated processes constituted by bone formation and bone resorption [1]. The formation of bone structures mainly depends upon the proliferation and differentiation of osteoblasts, which synthesize and deposit the mineralizing extracellular matrix of bone [2]. A number of growth factors and endocrinal hormones are involved in the differentiation of osteoblast, including bone morphogenetic proteins (BMPs) [3], Wnt [4], parathyroid hormone (PTH) [5], and insulinlike growth factors (IGFs) [6].

Increasing evidence indicates that bone is also under the control of immune and neuronal systems, besides the classical signaling pathways [7-9]. Mikihito and colleagues uncovered the essential role of Semaphorin 3A (Sema3A, original described as axon guidance factors in the central nervous system)-Neuropilin-1 (Nrp1) axis in the bone homeostasis maintenance [10]. Sema3A secreted by osteoblast exerts an osteoprotective effect by suppressing osteoclastic bone resorption and increasing osteoblastic bone formation. The binding of Sema3A to Nrp1 inhibits receptor activator of nuclear factor- $\kappa B$ ligand (RANKL)-induced osteoclast differentiation by suppressing the immunoreceptor tyrosine-based activation motif (ITAM) and RhoA signaling pathways. In addition, Sema3A-Nrp1 axis promotes osteoblast differentiation through the canonical Wnt/ $\beta$-catenin signaling pathway [10]. However, Fukuda and colleagues demonstrated that Sema3A regulates bone-mass accrual through sensory innervations indirectly, but not as directly as described by Mikihito [11].

Nrp1, co-receptor for the class 3 semaphorins (Sema3) and vascular endothelial growth factor (VEGF), transmits signals by forming a complex with other receptors due to its un-functional cytoplasmic domain [12]. For example, Nrp1 binds with TGF- $\beta$ receptor, forming complex to augment the canonical Smad2/3 signaling [13]. Nrp1 interacts with PDGF receptor $\alpha$ (PDGFR $\alpha$ ) and enhances PDGF stimulation of vascular smooth muscle cells (VSMCs) migration [14]. Nrp1 interacts with the EGF receptor (EGFR) or hepatocyte growth factor (HGF) to promote the signaling cascades elicited upon EGF or HGF stimulation [15-17]. To sum up, Nrp1 binds various types of ligands and co-receptors, and participates in multiple types of signaling pathways that control cell migration, survival, and attraction. However, whether Nrp1 plays essential roles in osteoblast differentiation and bone formation remains unclear.

Discoidin domain receptor 2 (DDR2), which was named after the discovery of discoidin homology in its extracellular region, belongs to the receptor tyrosine kinase (RTK) family and functions as collagen receptor [18]. Collagen binds with DDR2 via the discoidin (DS) domain of DDR2, leading to the slow-on and slow-off activation of DDR2 $[18,19]$. Ddr2 knockout mice and slie mice (mouse colony with spontaneous autosomal-recessive mutation in the Ddr2 locus) exhibit short long bones and irregular growth of flat bones [20]. Human Ddr2 gene mutation causes a rare form of dwarfism, spondylo-meta-epiphyseal dysplasia short limbhand type (SMED-SL) [21-23]. Our and other's studies also demonstrated that DDR2 plays essential roles in osteoblast differentiation and chondrocyte maturation by modulating the phosphorylation and transactivity of Runx2 in an ERK MAPK-dependent manner [24, 25].

Both Nrp1 and DDR2 are DS domain related proteins because of the DS domain in their extracellular domains $[26,27]$. Due to the similar structural characteristics, Nrp1 has functions in common with DDR2, such as roles in angiogenesis [28-30], teeth formation [31, $32]$, wound healing [33, 34], and female reproductive cycle [35-37]. In this study, we aimed to examine the function of Nrp1 in osteoblast differentiation, and to test whether there is a close correlation between Nrp1 and DDR2.

\section{Materials and Methods}

Antibodies and reagents

Goat anti-DDR2 (R\&D Systems, MN, USA), rabbit anti-Nrp1, mouse anti- $\beta$-actin (Abcam, MA, USA), goat anti-Runx2 (Santa Cruz, TEX, USA), rabbit anti-ERK1/2 (Cell Signaling Technology, MA, USA). BMP-2 (Peprotech, NJ, USA), Ascorbic acid (AA), $\beta$-glycerophosphate ( $\beta$-GP) (Sigma, IL, USA).

\section{KARGER}




\section{Cellular Physiology Cell Physiol Biochem 2015;36:75-84

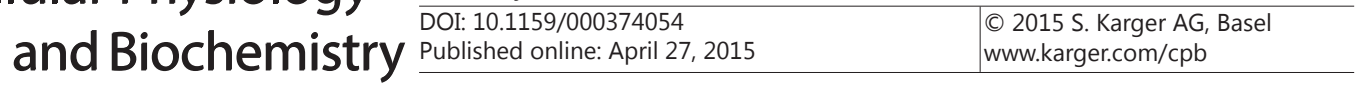 \\ Zhang et al.: Nrp1 Augments DDR2-Mediated Signaling in Osteoblastogenesis}

Cell cultures

Murine preosteoblast MC3T3-E1 subclone 14 cells (ATCC, VA, USA) were cultured in $\alpha$-MEM (Invitrogen, CA, USA) containing 10\% FBS. Murine myoblast C2C12 (ATCC) were cultured in DMEM containing 10\% FBS. To induce osteogenic differentiation, cells were induced by osteogenic inducers: $50 \mu \mathrm{g} / \mathrm{mL}$ AA, $5 \mathrm{mM} \beta$-GP for MC3T3-E1 cells and $200 \mathrm{ng} / \mathrm{mL}$ of BMP-2 for C2C12 cells. The medium was replaced every other day unless otherwise indicated.

\section{Cell transfection and infection}

For the plasmid transfection, construct containing Nrp1 was transfected to cells at the concentration of $400 \mathrm{ng} / \mathrm{cm}^{2}$. For the transfection of small interfering RNAs (siRNAs), $200 \mathrm{nM}$ of siRNA targeting mouse Nrp1 gene was introduced into cells. For the lentivirus infection, cells with $50 \%$ confluence were incubated with virus-containing medium for 48 hours and further grown in medium containing $4 \mathrm{mg} / \mathrm{mL}$ of puromycin (Invitrogen, CA, USA). Fresh selective medium was added every other day. After 3 weeks of culture, single colonies were picked up, expanded, and maintained.

\section{Determination of osteogenic differentiation}

MC3T3-E1 cells were induced with osteogenic inducers. At the designated time points, cells were collected for analyzing of mRNA levels of osteogenic markers. ALP activity assays were performed using a commercial kit (Nanjing Jiancheng Bioengineering Institute, Nanjing, P.R.C) in accordance with the manufacturer's instructions. Protein extract concentrations were measured using BCA kit (Pierce, IL, USA) to normalize the relative ALP activity. The amount of OCN in cell supernatants was detected using a commercially available ELISA kit (R\&D Systems).

\section{Real-time PCR}

Total RNA was extracted using Trizol (Invitrogen). Complementary DNA (cDNA) was synthesized from $2 \mu \mathrm{g}$ of total RNA using Super-Script II First-Strand Synthesis System (Invitrogen). Quantitative real-time PCR was performed using $200 \mathrm{nM}$ of each primer in CFX96 Sequence Detection System (Bio-Rad, CA, USA). Comparative threshold cycle $(\mathrm{Ct})$ method was used for relative quantification.

\section{Western blotting}

Cell lysates were resolved by 10\% SDS-polyacrylamide gel electrophoresis (SDS-PAGE) and transfered to Hybond-ECL nitrocellulose membranes (Amersham Biosciences, NJ, USA). After being blocked in Trisbuffered saline containing $0.1 \%$ Tween- 20 at room temperature for 1 hour, membranes were incubated with the indicated primary antibodies at $4{ }^{\circ} \mathrm{C}$ overnight. Then the membranes were further incubated with corresponding horseradish peroxidase (HRP)-conjugated secondary antibodies at room temperature for 1 hour. Immunoblots of protein bands were visualized with an enhanced chemiluminescence (ECL) detection kit (Amersham Life Sciences, Piscataway, NJ, USA).

Immunofluorescent assay

Cells were fixed in $4 \%$ paraformaldehyde, then were permeabilized with $0.1 \%$ Triton X- 100 . Permeabilized cells were washed and blocked with 10\% goat serum for 20 min. After washing with PBS, cells were incubated with primary antibody at $4{ }^{\circ} \mathrm{C}$ overnight, followed by incubation of TRITC-conjugated anti-goat or FITC-conjugated anti-rabbit antibody at room temperature for 1 hour. Nuclei was stained by 4', 6-diamidino-2-phenylindole (DAPI).

\section{Statistical analyses}

Data are expressed as the mean \pm SD of three independent experiments. The difference between two groups was analyzed by student's T-test using the GraphPad Prism software version 4.0. A value of $\mathrm{p}<0.05$ was considered statistically significant.

\section{Results}

Expression profiles of Nrp1 paralleled with that of DDR2 during osteoblast differentiation In our previous study, we implicated the essential role of DDR2 in osteoblast differentiation and demonstrated the involvement of ERK-Runx2 cascade in the DDR2mediated signaling. To find out whether Nrp1 plays role in osteoblast differentiation, we 
tested the expression profile of Nrp1. MC3T3-E1 cells were induced and the mRNA levels of osteoblastic markers, including ALP, Runx2, Osterix and OCN, were examined at the indicated durations. As shown in Fig. 1A, the mRNA levels of ALP Runx2, Osterix, and OCN were elevated significantly and sequentially, suggesting the successfully established differentiation models. Both the mRNA levels of Nrp1 and DDR2 peaked at day 3 and 6 and then undergone a gradual decrease to the basal levels (Fig. 1B). Immunoblot was performed to detect the protein changes. As demonstrated Fig. 1C and D, the protein levels of Nrp1 and DDR2 were consistent with the results of mRNA analysis. To further validate the involvement of Nrp1 in osteoblast differentiation, $\mathrm{C} 2 \mathrm{C} 12$, another classical osteoblast precursors, were induced and harvested for mRNA and protein analysis. As expected, the C2C12 differentiation model was successfully established (Fig. 1E) and the change of Nrp1 expression also showed a similar trend compared with that of DDR2 (Fig. 1F-H). Collectively, these results suggested that the expression profile of Nrp1 paralleled with that of DDR2 during osteoblast differentiation and Nrp1 is probably involved in osteoblast differentiation.

\section{Nrp1 stimulated DDR2-induced osteoblast differentiation}

To find out the biological meaning of the Nrp1-DDR2 paralleled expression pattern and define the roles of Nrp1 in osteoblast differentiation, Nrp1 was stably overexpressed or knocked down in MC3T3-E1 cells or DDR2-modificated MC3T3-E1 cells. As confirmed in Fig. 2A, the expression levels of Nrp1 and DDR2 were efficiently modified after lentivirusmediated gene transduction. Control and genetically modified cells were cultured in the presence or absence of differentiation inducers for 12 days, and osteogenic indicators were analyzed. Real-time PCR results showed that overexpression of Nrp1 led to dramatic

Fig. 1. Nrp1 expression paralleled with that of DDR2 during osteogenic differentiation. (A) Verification of MC3T3-E1 differentiation. (B) The mRNA expression of Nrp1 and DDR2 during MC3T3-E1 differentiation. (C) The protein level of Nrp1 and DDR2 during MC3T3-E1 differentiation. (D) The quantification of protein levels of Nrp1 and DDR2 in MC3T3-E1 cells. (E) Verification of $\mathrm{C} 2 \mathrm{C} 12$ differentiation. (F) The mRNA expression of Nrp1 and DDR2 during $\mathrm{C} 2 \mathrm{C} 12$ differentiation. (G) Immunoblot analysis of Nrp1 and DDR2 protein expression during C2C12 differentiation. (H) The quantification of protein levels of Nrp1 and DDR2 in C2C12 cells.

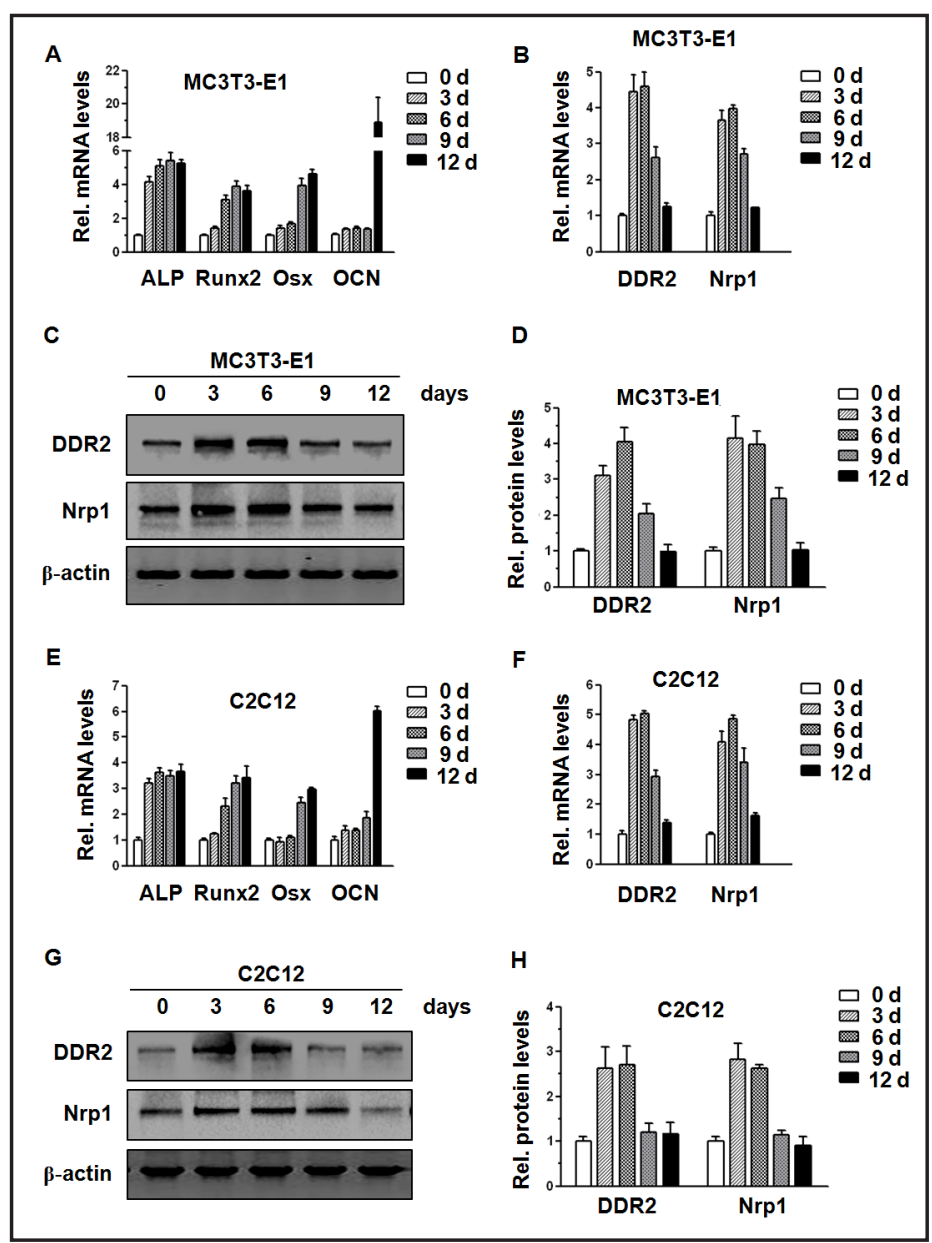


Fig. 2. Nrp1 stimulated DDR2-induced osteoblast differentiation. (A) The efficiency of overexpression of Nrp1 and DDR2. (B) Nrp1 overexpression increased the mRNA levels of osteoblastic markers induced by DDR2, whereas Nrp1 knockdown decreased the DDR2-induced mRNA expression of osteoblastic markers. (C) Nrp1 enhanced the DDR2-inducted ALP activity. (C) Nrp1 facilitated the DDR2-inducted OCN production. ${ }^{*} \mathrm{p}<0.05$ versus DDR2-overexpression group.

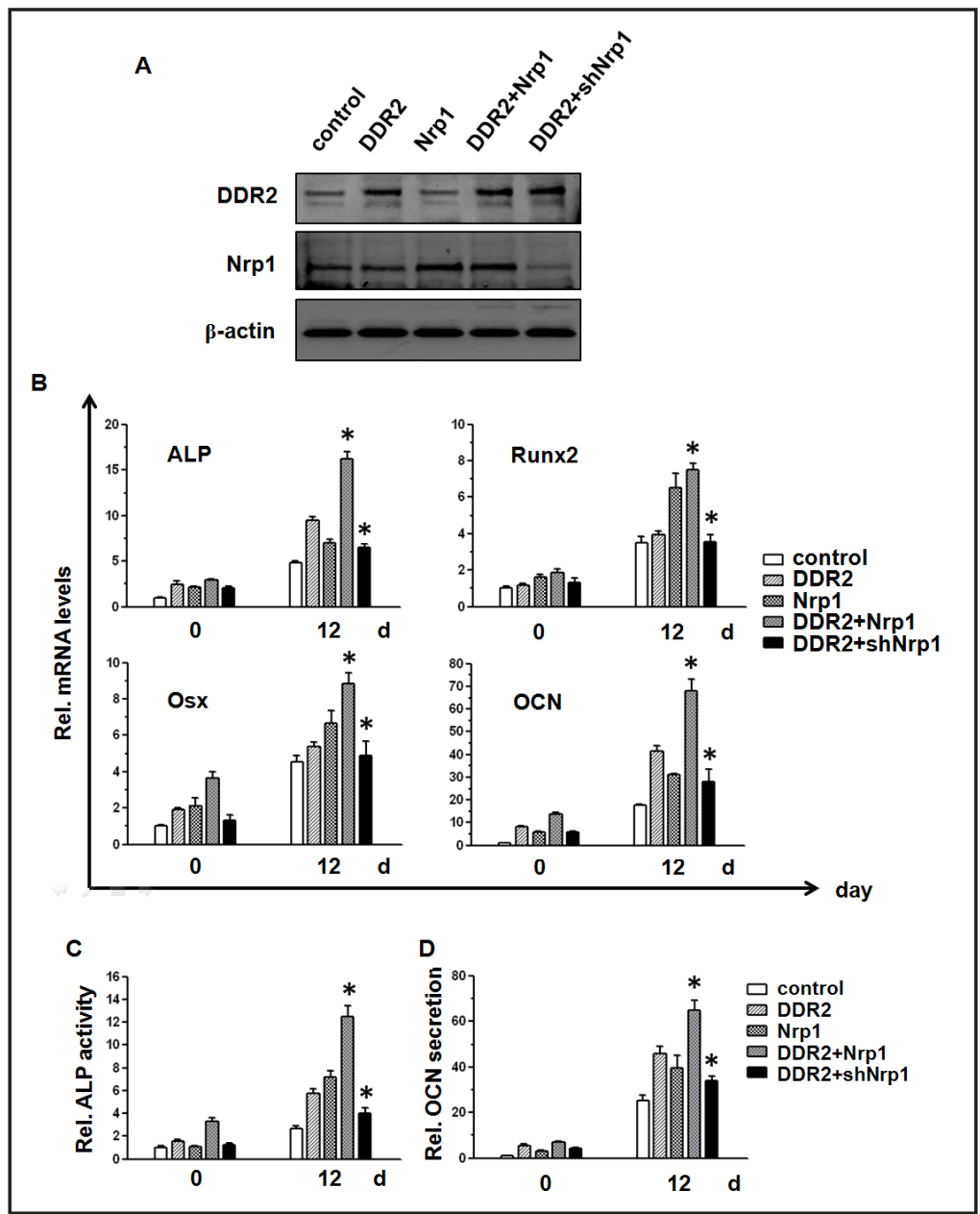

Fig. 3. Nrp1 enhanced the DDR2-mediated signaling in osteoblast differentiation. (A) Immunoblot analysis of phosphorylation of DDR2, ERK1/2, and Runx2. (B) The quantification of the ratio of phosphorylated DDR2 to total DDR2. (C) The quantification of the ratio of phosphorylated ERK1/2 to total ERK1/2. (D) The quantification of the ratio of phosphorylated Runx2 to total Runx2.

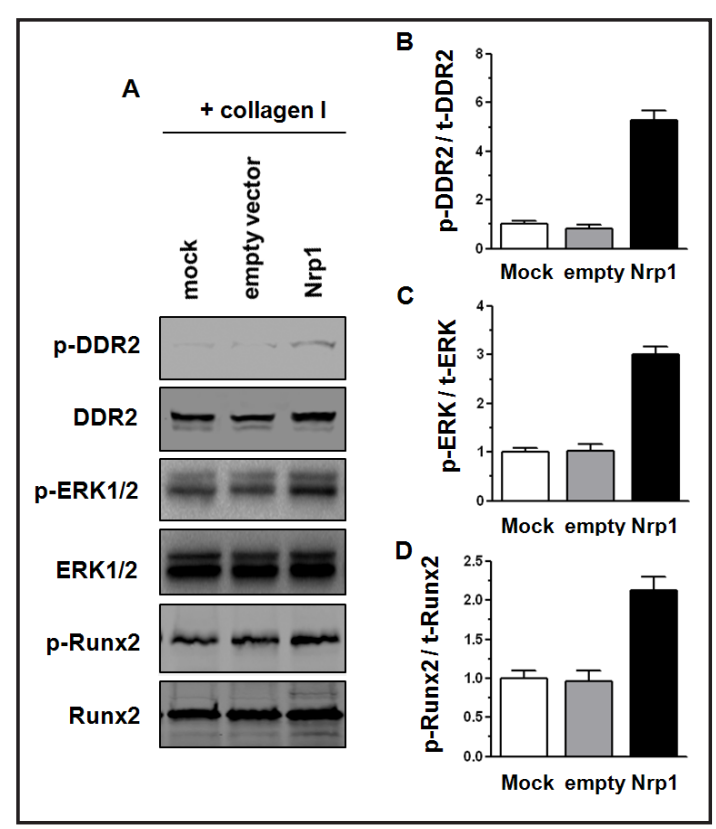

increase in mRNA levels of osteoblastic markers, as similar as DDR2 overexpression. When Nrp1 was introduced in the DDR2-overexpressing cells, the elevation of mRNA expression of osteoblastic markers were significantly augmented, compared with the without-Nrp1 
Fig. 4. Nrp1 stabilized DDR2 and inhibited its degradation. (A) The co-occurrence of Nrp1 and DDR2 in MC3T3-E1 cells. The MC3T3-E1 cells were cultured in presence of osteogenic inducer, 6 days later cells were fixed for immunofluorescent detection. Scale bars $=20 \mu \mathrm{m}$. (B) Over-expression of Nrp1 did not change the mRNA transcript of DDR2. (C) Knockdown of Nrp1 did not alter the mRNA expression of DDR2. (D) Over-expression of Nrp1 increased the abundance of DDR2. MC3T3-E1 cells were mock treated or transfected with Nrp1 containing plasmid, 24 hours later cells were treated with $100 \mu \mathrm{M}$ emetine for the indicated durations, and DDR2 abundance was examined. (E) Relative levels of DDR2 to $\beta$-actin were quantified.

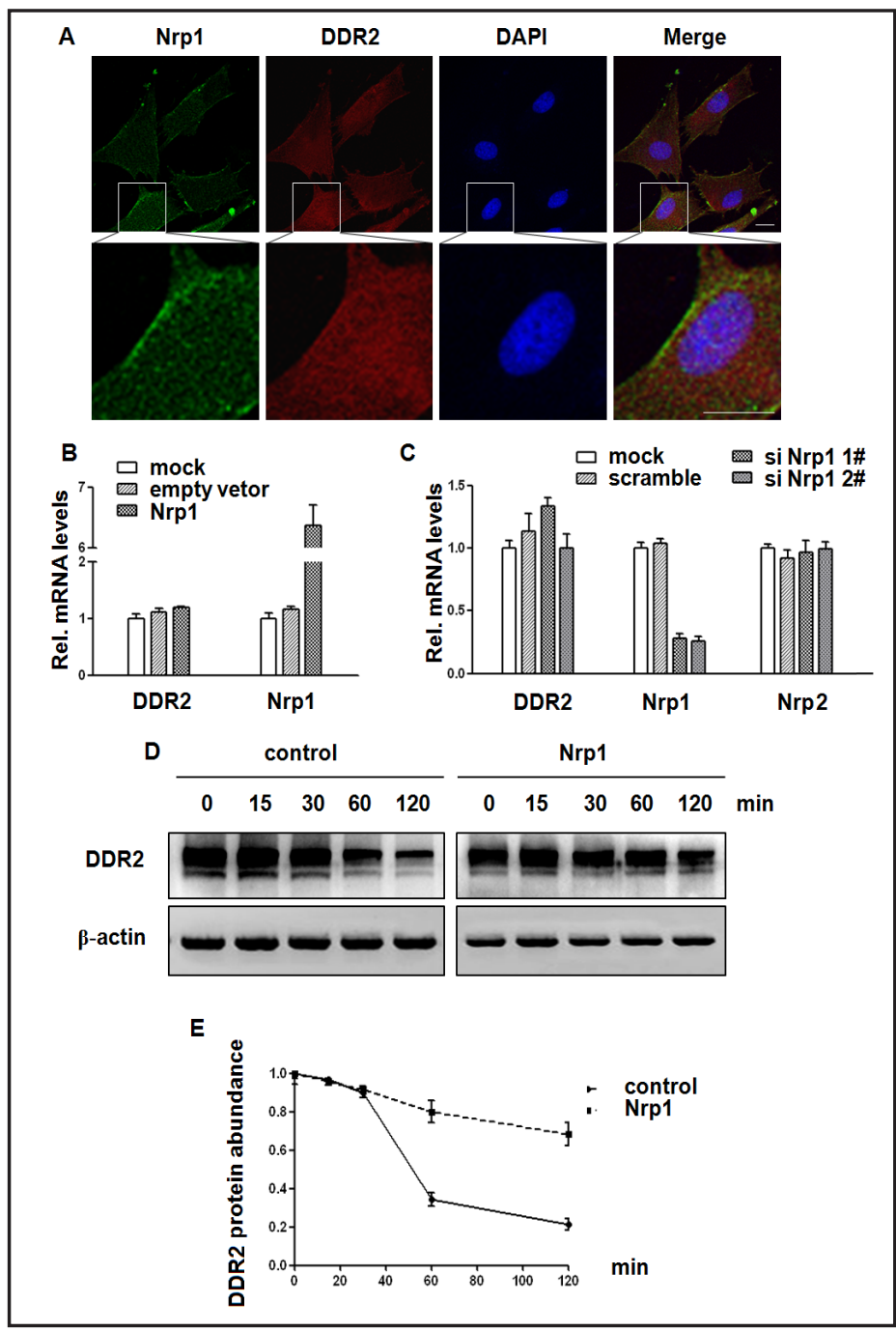

controls. In contrast, when Nrp1 was depleted in the DDR2-overexpressing cells, the elevation of mRNA expression was significantly dropped (Fig. 2B). Consistent with the mRNA results, ALP activity and the secretion of OCN also displayed a similar change (Fig. 2C and D). Taken together, these results demonstrated that Nrp1 stimulated the DDR2-mediated osteoblast differentiation.

Nrp1 enhanced the DDR2-mediated signaling in osteoblast differentiation

To investigate the molecular mechanism that Nrp1 facilitated DDR2-induced osteoblast differentiation, we tried to find out whether DDR2-induced signaling was affected after Nrp1 expression was modified. Our previous study demonstrated that DDR2 activates Runx2 in an ERK-dependent manner. With the increase of collagen type I expression, a characteristic of osteoblast differentiation, the phosphorylation of DDR2, ERK1/2, and Runx2 was induced [24]. Therefore, we analyzed the phosphorylation of Runx2 and ERK1/2 to detect the effect of Nrp1 on DDR2-induced downstream signaling. We transfected MC3T3-E1 cells with empty vector or vector containing Nrp1 cDNA, 24 hours later the cells were seeded on collagen I-coated plates for 6 hour before immunoblot was performed. As shown in Fig. 3A and B, in Nrp1-overexpressing MC3T3-E1 cells, the expression and phosphorylation of DDR2 was significantly elevated. As expected, Nrp1 overexpression also led to significant increase in phophorylated ERK1/2 and Runx2 levels (Fig. 3A, C, and D). These results suggested that Nrp1 amplified the stimulatory role of DDR2 in osteoblast differentiation through enhancing the DDR2-induced phosphorylation of ERK1/2 and Runx2.

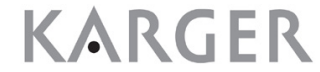


Nrp1 stabilized DDR2 and inhibited its degradation

Given the paralleled expression pattern of Nrp1 and DDR2, and the stimulatory effect of Nrp1 on DDR2-mediated signaling, we detected the cellular localization of Nrp1 and DDR2 by performing immunofluorescent assay. MC3T3-E1 cells were induced for 6 days before being used to perform immunofluorescent analysis. As shown in Fig. 4A, DDR2 located in the plasma and cytoplasm, and Nrp1 mainly located in the plasma. A large portion of DDR2 and Nrp1 were found co-located in the plasma membrane.

To find out how Nrp1 regulates the activation of DDR2 and downstream effectors, we examined the effect of Nrp1 on DDR2 expression. MC3T3-E1 cells were transfected with empty vector or vector containing Nrp1 cDNA, and 48 hour later cells were harvested for mRNA analysis. As shown in Fig. 4B, the mRNA levels of DDR2 were not changed after Nrp1 was overexpressed. Consistently, the deficiency of Nrp1 induced by specific siRNA did not alter the levels of DDR2 mRNA (Fig. 4C). Given the increase in DDR2 protein level in Nrp1overexpressing cells (Fig. 3A), and the abundance of protein could be regulated by changes in the rate of synthesis or degradation, we hypothesized that Nrp1 may exert a modification on the degradation of DDR2. To test this hypothesis, we used emetine, a protein synthesis inhibitor, to examine the DDR2 abundance and stability. As shown in Fig. 4D and E, DDR2 protein began to degrade obviously in control cells at the time point of $60 \mathrm{~min}$. At $120 \mathrm{~min}$, the protein abundance of DDR2 dropped significantly to approximate twenty percent of that of control cells. However, we detected significantly high expression levels of DDR2 protein in Nrp1-overexpressing cells, as compared with that in controls. Until 120 min post-emetine treatment, there was no obvious decrease in DDR2 protein level. Collectively, these results demonstrated that Nrp1 did not alter the transcript of DDR2, but could inhibit the natural degradation and prolonged the half-life of DDR2.

\section{Discussion}

In the present study, we demonstrated that Nrp1 expression paralleled with that of DDR2 during osteoblast differentiation, which brought the possibility of their correlated function. Further functional experiments revealed that Nrp1 assisted the promoting role of DDR2 in osteoblast differentiation, via activation of DDR2-mediated downstream signaling. Evidence from immunofluorescent confirmed the co-occurrence of Nrp1 and DDR2. Modification of Nrp1 expression did not change the mRNA transcript of DDR2, but Nrp1 overexpression prolonged the half-life of DDR2 protein. Our results demonstrated the stimulatory role of Nrp1 in DDR2-induced osteoblast differentiation for the first time, providing molecular evidence for exploiting Nrp1 and DDR2 as novel targets to treat bone-related disease.

The structural similarity of Nrp1 and DDR2 is that they are both single-pass membrane receptors containing DS domain, which is a $\sim 150$ amino acid motif involved in molecular and/or cellular interactions. DS domain is composed of eight $\beta$-strands arranged in a compact $\beta$-barrel and three finger-like loops called "spikes" forming ligand recognition/ binding surface [27]. The "spike" 1 and 3 of DDR2 DS domain are responsible for binding collagen [18], while the three "spikes" in the first DS domain of Nrp1 correspond to Sema3s binding regions [12]. In our study, we demonstrated the co-occurrence of Nrp1 and DDR2, and this co-occurrence depends upon the stage of cell differentiation, since we only detected it in differentiated osteoblast. The difference in localization between un- and differentiated conditions was probably due to the distinct extracellular differentiation stimuli. We hypothesized that it is collagen I, whose expression level increases during osteoblast differentiation, that bridges Nrp1 and DDR2 together through binding DS domains. We intend to investigate the involvement of collagen I in the near future. Further experiments are also needed to fully confirm the Nrp1-DDR2 interaction and the detailed structural basis.

Nrp1 is co-receptor of VEGF165 for cooperating with VEGFR to transmit pro-angiogenic signals [12]. Our previous study suggested that DDR2 promotes proliferation, migration and tube formation of primary human umbilical vein endothelial cells (HUVECs), suggesting 


\section{Cellular Physiology Cell Physiol Biochem 2015;36:75-84 \begin{tabular}{l|l|l} 
DOI: 10.1159/000374054 2015 & O 2015 S. Karger AG, Basel \\
and Biochemistry
\end{tabular}

the critical role of DDR2 in angiogenesis [30]. The present study implicated the synergistic role of Nrp1 in DDR2-induced osteoblastogenesis. Together with other commence in teeth formation [31, 32], wound healing [33, 34], and regulation of reproductive cycle [35-37], it could be deduced that DDR2 and Nrp1 are not only structurally but also functionally related proteins. It is probably that DDR2 also forms complex with Nrp1 and this two molecules act in concert with each other in other aspects.

The cytoplasmic domain of Nrp1 lacks functional signaling motif, thus calling for binding with other receptor(s) to transmit signals. In bone formation and resorption, Sema3A-Nrp1 employs PlexA1 to activate downstream signaling. Whether PlexA1 is involved in the Nrp1 regulation of DDR2 remains to be further investigated.

The importance of DDR2 in osteoblast differentiation and bone formation has been indicated by our group and other's [24, 25]. Loss of function of human or mouse Ddr2 gene leads to dysplasia in bones [20-23]. Therefore, the regulation of DDR2 in osteoblast differentiation is of great importance. The abundance of receptors is negatively regulated by internalization and degradation, which are necessary to avoid the over-activation of downstream signaling. In our study, we found that Nrp1 expression did not affect the mRNA transcript of DDR2, but modulated its protein abundance. Half-life determination assay demonstrated that Nrp1 stabilized DDR2. It is possible that Nrp1 stabilized DDR2 by blocking the binding site for ubiquitin or other regulatory protein (such as lysosomal enzymes involved in lysosomal pathway) and suppressing the negative regulation of DDR2.

Taken together, our results show for the first time that Nrp1 functions as an important player in the DDR2-mediated bone protection network. This finding enriches the current knowledge of DDR2 and Nrp1, and provides strategy for exploiting Nrp1 and DDR2 as promising targets for the treatment of bone-related deseased.

\section{Disclosure Statement}

The authors state that they have no conflicts of interest.

\section{Acknowledgments}

This work is supported by National Natural Science Foundation of China (No. 81300716), Specialized Research Fund for the Doctoral Program of Higher Education (No. 20120201120089) and Scientific Research Fund of Shaanxi Provincial Department of Health (No. 2012D59).

\section{References}

1 Raggatt LJ, Partridge NC: Cellular and molecular mechanisms of bone remodeling. J Biol Chem 2010;285:25103-25108.

-2 Fakhry M, Hamade E, Badran B, Buchet R, Magne D: Molecular mechanisms of mesenchymal stem cell differentiation towards osteoblasts. World J Stem Cells 2013;5:136-148.

-3 Kopf J, Paarmann P, Hiepen C, Horbelt D, Knaus P: BMP growth factor signaling in a biomechanical context. Biofactors 2013;40:171-187

-4 Regard JB, Zhong Z, Williams BO, Yang Y: Wnt signaling in bone development and disease: making stronger bone with Wnts. Cold Spring Harb Perspect Biol 2012;4(12).

5 Ezura Y, Noda M: Parathyroid hormone: its anabolic action on bone. Clin Calcium 2013;23:203-209.

6 Guntur AR, Rosen CJ: IGF-1 regulation of key signaling pathways in bone. Bonekey Rep 2013;2:437.

7 Lange U, Teichmann J, Schett G, Neumann E, Müller-Ladner U: Osteoimmunology: how inflammation influences bone metabolism. Dtsch Med Wochenschr. 2013;138:1845-1849.

8 Takeda S: Intercommunication between bone and central nervous system. Clin Calcium 2013;23:173-179. 


\section{Cellular Physiology Cell Physiol Biochem 2015;36:75-84 \begin{tabular}{c|l|}
\cline { 2 - 2 } DOI: 10.1159/000374054 2015 & O $2015 \mathrm{~S}$. Karger AG, Basel \\
\hline and Biochemistry
\end{tabular} \\ Zhang et al.: Nrp1 Augments DDR2-Mediated Signaling in Osteoblastogenesis}

9 Colaianni G, Cuscito C, Colucci S: FSH and TSH in the regulation of bone mass: the pituitary/immune/bone axis. Clin Dev Immunol 2013; 2013:382698.

10 Hayashi M, Nakashima T, Taniguchi M, Kodama T, Kumanogoh A, Takayanagi H: Osteoprotection by semaphorin 3A. Nature 2012;485:69-74.

11 Fukuda T, Takeda S, Xu R, Ochi H, Sunamura S, Sato T, Shibata S, Yoshida Y, Gu Z, Kimura A, Ma C, Xu C, Bando W, Fujita K, Shinomiya K, Hirai T, Asou Y, Enomoto M, Okano H, Okawa A, Itoh H: Sema3A regulates bone-mass accrual through sensory innervations. Nature 2013;497:490-493.

12 Pellet-Many C, Frankel P, Jia H, Zachary I: Neuropilins: structure, function and role in disease. Biochem J 2008;411:211-226.

13 Glinka Y, Stoilova S, Mohammed N, Prud'homme GJ: Neuropilin-1 exerts co-receptor function for TGFbeta- 1 on the membrane of cancer cells and enhances responses to both latent and active TGF-beta. Carcinogenesis 2011;32:613-621.

14 Pellet-Many C, Frankel P, Evans IM, Herzog B, Jünemann-Ramírez M, Zachary IC: Neuropilin-1 mediates PDGF stimulation of vascular smooth muscle cell migration and signalling via p130Cas. Biochem J 2011;435:609-618.

15 Hu B, Guo P, Bar-Joseph I, Imanishi Y, Jarzynka MJ, Bogler O, Mikkelsen T, Hirose T, Nishikawa R, Cheng SY: Neuropilin-1 promotes human glioma progression through potentiating the activity of the HGF/SF autocrine pathway. Oncogene 2007;26:5577-5586.

16 Rizzolio S, Rabinowicz N, Rainero E, Lanzetti L, Serini G, Norman J, Neufeld G, Tamagnone L: Neuropilin-1dependent regulation of EGF-receptor signaling. Cancer Res 2012;72:5801-5811.

-17 Xu R, Li Q Zhou J, Zhou X, Perelman JM, Kolosov VP. Secretoneurin induces airway mucus hypersecretion by enhancing the binding of EGF to NRP1. Cell Physiol Biochem. 2014;33:446-56.

$\checkmark 18$ Vogel W: Discoidin domain receptors: structural relations and functional implications. FASEB J 1999;13:S77-82.

19 Vogel WF, Abdulhussein R, Ford CE: Sensing extracellular matrix: an update on discoidin domain receptor function. Cell Signal 2006;18:1108-1116.

20 Kano K, Marín de Evsikova C, Young J, Wnek C, Maddatu TP, Nishina PM, Naggert JK: A novel dwarfism with gonadal dysfunction due to loss-of-function allele of the collagen receptor gene, Ddr2, in the mouse. Mol Endocrinol 2008;22:1866-1880.

-21 Ali BR, Xu H, Akawi NA, John A, Karuvantevida NS, Langer R, Al-Gazali L, Leitinger B: Trafficking defects and loss of ligand binding are the underlying causes of all reported DDR2 missense mutations found in SMED-SL patients. Hum Mol Genet 2010;19:2239-2250.

22 Bargal R, Cormier-Daire V, Ben-Neriah Z, Le Merrer M, Sosna J, Melki J, Zangen DH, Smithson SF, Borochowitz Z, Belostotsky R, Raas-Rothschild A: Mutations in DDR2 gene cause SMED with short limbs and abnormal calcifications. Am J Hum Genet 2009;84:80-84.

23 Al-Kindi A, Kizhakkedath P, Xu H, John A, Sayegh AA, Ganesh A, Al-Awadi M, Al-Anbouri L, Al-Gazali L, Leitinger B, Ali BR: A novel mutation in DDR2 causing spondylo-meta-epiphyseal dysplasia with short limbs and abnormal calcifications (SMED-SL) results in defective intra-cellular trafficking. BMC Med Genet 2014;15:42.

24 Zhang Y, Su J, Yu J, Bu X, Ren T, Liu X, Yao L: An essential role of discoidin domain receptor 2 (DDR2) in osteoblast differentiation and chondrocyte maturation via modulation of Runx2 activation. J Bone Miner Res 2011;26:604-617.

25 Lin KL, Chou CH, Hsieh SC, Hwa SY, Lee MT, Wang FF: Transcriptional upregulation of DDR2 by ATF4 facilitates osteoblastic differentiation through p38 MAPK-mediated Runx2 activation. J Bone Miner Res 2010;25:2489-24503.

26 Geretti E, Shimizu A, Klagsbrun M: Neuropilin structure governs VEGF and semaphorin binding and regulates angiogenesis. Angiogenesis 2008;11:31-39.

27 Kiedzierska A, Smietana K, Czepczynska H, Otlewski J: Structural similarities and functional diversity of eukaryotic discoidin-like domains. Biochim Biophys Acta 2007;1774:1069-1078.

28 Koch S: Neuropilin signalling in angiogenesis. Biochem Soc Trans 2012;40:20-25.

29 Chen C, Li M, Chai H, Yang H, Fisher WE, Yao Q: Roles of neuropilins in neuronal development, angiogenesis, and cancers. World J Surg 2005;29:271-275.

30 Zhang S, Bu X, Zhao H, Yu J, Wang Y, Li D, Zhu C, Zhu T, Ren T, Liu X, Yao L, Su J: A host deficiency of discoidin domain receptor 2 (DDR2) inhibits both tumour angiogenesis and metastasis. J Pathol 2014;232:436-448. 


\section{Cellular Physiology Cell Physiol Biochem 2015;36:75-84 \begin{tabular}{l|l|} 
DOI: 10.1159/000374054 & O 2015 S. Karger AG, Basel
\end{tabular} \\ Zhang et al.: Nrp1 Augments DDR2-Mediated Signaling in Osteoblastogenesis}

-31 Sijaona A, Luukko K, Kvinnsland IH, Kettunen P: Expression patterns of Sema3F, PlexinA4, -A3, Neuropilin1 and -2 in the postnatal mouse molar suggest roles in tooth innervation and organogenesis. Acta Odontol Scand 2012;70:140-148.

-32 Løes S, Kettunen P, Kvinnsland IH, Taniguchi M, Fujisawa H, Luukko K: Expression of class 3 semaphorins and neuropilin receptors in the developing mousetooth. Mech Dev 2001;101:191-194.

-33 Nagy N, Németh IB, Szabad G, Szolnoky G, Belsõ N, Bata-Csörgõ Z, Dobozy A, Kemény L, Széll M: The altered expression of syndecan 4 in the uninvolved skin of venous leg ulcer patients may predispose to venous leg ulcer. Wound Repair Regen 2008;16:495-502.

-34 Olaso E, Lin HC, Wang LH, Friedman SL: Impaired dermal wound healing in discoidin domain receptor 2-deficient mice associated with defective extracellular matrix remodeling. Fibrogenesis Tissue Repair 2011;4:5.

35 Stouffer RL, Martínez-Chequer JC, Molskness TA, Xu F, Hazzard TM: Regulation and action of angiogenic factors in the primate ovary. Arch Med Res 2001;32:567-575.

36 Matsumura H, Kano K, Marín de Evsikova C, Young JA, Nishina PM, Naggert JK, Naito K: Transcriptome analysis reveals an unexpected role of a collagen tyrosine kinase receptor gene, Ddr2, as a regulator of ovarian function. Physiol Genomics 2009;39:120-129.

-37 Giacobini P, Parkash J, Campagne C, Messina A, Casoni F, Vanacker C, Langlet F, Hobo B, Cagnoni G, Gallet S, Hanchate NK, Mazur D, Taniguchi M, Mazzone M, Verhaagen J, Ciofi P, Bouret SG, Tamagnone L, Prevot V: Brain endothelial cells control fertility through ovarian-steroid-dependent release of semaphorin 3A. PLoS Biol 2014;12:e1001808. 